\title{
Dynamic Observers for Fault Diagnosis of Timed Systems
}

\author{
Franck Cassez, Member, IEEE
}

\begin{abstract}
In this paper we extend the work on dynamic observers for fault diagnosis [1], [2], [3] to timed automata. We study sensor minimization problems with static observers and then address the problem of computing the most permissive dynamic observer for a system given by a timed automaton.
\end{abstract}

\section{INTRODUCTION}

Discrete-event systems [4] (DES) can be modelled by finite automata over an alphabet of actions/events $\Sigma$. The fault diagnosis problem [5] for DES consists in detecting faulty sequences in the system. A faulty sequence is a sequence of the DES containing an occurrence of a special event $f$. It is assumed that an external observer which has to detect faults, knows the specification/model of the DES, but can partially observe the system at runtime: it is able to observe sequences of observable events in $\Sigma_{o} \subseteq \Sigma$. Based on this knowledge, it has to announce whether an observation (in $\Sigma_{o}^{*}$ ) stems from a faulty sequence (in $(\Sigma \cup\{\tau, f\})^{*}$ ). Checking diagnosability of DES can be done in PTIME and computing a diagnoser amounts to determinizing the DES (EXPTIME) [5], [6], [7].

Fault Diagnosis for Timed Automata. The fault diagnosis problem for Timed Automata (TA) has been introduced and solved by $\mathrm{S}$. Tripakis in [8], where he proved that checking diagnosability of a timed automaton is PSPACEcomplete. In the timed case however, the diagnoser may be a Turing machine. In a subsequent work by P. Bouyer and F. Chevalier [9], the problem of checking whether a timed automaton is diagnosable using a diagnoser which is a deterministic timed automaton (DTA) was studied, and they proved that this problem was 2EXPTIME-complete.

Our Contribution and Related Work. In [1], [2] (and [3] for an extended version), we have introduced dynamic $o b$ servers for fault diagnosis of DES. In this framework, an observer can choose dynamically which events it is going to observe and make a new choice after each occurrence of any (currently) observable event. In [1], [3] we have shown how to compute (2EXPTIME) a most permissive observer which represents all the the dynamic observers that ensures that a DES is diagnosable. In [2] we have furthermore introduced a notion of cost of an observer, and proved that an optimal observer could also be computed in 2EXPTIME.

In this paper, we extend the previous results for systems given by timed automata. We first settle the complexity of some optimization problems with static observers

Franck Cassez is with National ICT Australia \& CNRS, Locked Bag 6016, The University of New South Wales, Sydney NSW 1466, Australia. franck.cassez@cnrs.irccyn.fr, Franck. Cassez@nicta.com.au

Author supported by a Marie Curie International Outgoing Fellowship within the 7th European Community Framework Programme. (section IV). We then focus on dynamic timed observers, and show how to compute (section V) a most permissive (timed) dynamic observer, under the assumption of bounded resources. In section $\mathrm{VI}$, we define a notion of cost for timed observers (which extends the one we have defined for DES in[2]) and show how to compute the cost of a given observer. We also discuss the problem of synthesizing an optimal timed dynamic observer.

\section{PRELIMINARIES}

$\Sigma$ denotes a finite alphabet and $\Sigma_{\tau}=\Sigma \cup\{\tau\}$ where $\tau \notin \Sigma$ is the unobservable action. $\mathbb{B}=\{$ TRUE, FALSE $\}$ is the set of boolean values, $\mathbb{N}$ the set of natural numbers, $\mathbb{Z}$ the set of integers and $\mathbb{Q}$ the set of rational numbers. $\mathbb{R}$ is the set of real numbers and $\mathbb{R}_{\geq 0}$ is the non-negative real numbers.

\section{A. Clock Constraints}

Let $X$ be a finite set of variables called clocks. A clock valuation is a mapping $v: X \rightarrow \mathbb{R}_{\geq 0}$. We let $\mathbb{R}_{\geq 0}^{X}$ be the set of clock valuations over $X$. We let $\mathbf{0}_{X}$ be the zero valuation where all the clocks in $X$ are set to 0 (we use $\mathbf{0}$ when $X$ is clear from the context). Given $\delta \in \mathbb{R}, v+\delta$ denotes the valuation defined by $(v+\delta)(x)=v(x)+\delta$. We let $\mathcal{C}(X)$ be the set of convex constraints on $X$, i.e., the set of conjunctions of constraints of the form $x \bowtie c$ with $c \in \mathbb{Z}$ and $\bowtie \in\{\leq,<,=,>, \geq\}$. Given a constraint $g \in \mathcal{C}(X)$ and a valuation $v$, we write $v \mid=g$ if $g$ is satisfied by $v$. Given $R \subseteq X$ and a valuation $v, v[R]$ is the valuation defined by $v[R](x)=v(x)$ if $x \notin R$ and $v[R](x)=0$ otherwise.

\section{B. Timed Words}

The set of finite (resp. infinite) words over $\Sigma$ is $\Sigma^{*}$ (resp. $\Sigma^{\omega}$ ) and we let $\Sigma^{\infty}=\Sigma^{*} \cup \Sigma^{\omega}$. We let $\varepsilon$ be the empty word. A language $L$ is any subset of $\Sigma^{\infty}$. A finite (resp. infinite) timed word over $\Sigma$ is a word in $\left(\mathbb{R}_{\geq 0} \cdot \Sigma\right)^{*} \cdot \mathbb{R}_{\geq 0}$ (resp. $\left.\left(\mathbb{R}_{\geq 0} . \Sigma\right)^{\omega}\right)$. $\operatorname{Dur}(w)$ is the duration of a timed word $w$ which is defined to be the sum of the durations (in $\mathbb{R}_{\geq 0}$ ) which appear in $w$; if this sum is infinite, the duration is $\infty$. Note that the duration of an infinite word can be finite, and such words which contain an infinite number of letters, are called Zeno words.

$T W^{*}(\Sigma)$ is the set of finite timed words over $\Sigma, T W^{\omega}(\Sigma)$, the set of infinite timed words and $T W(\Sigma)=T W^{*}(\Sigma) \cup$ $T W^{\omega}(\Sigma)$. A timed language is any subset of $T W(\Sigma)$.

In this paper we write timed words as $0.4 a 1.0 \mathrm{~b} 2.7 \mathrm{c}$. where the real values are the durations elapsed between two letters: thus $c$ occurs at global time 4.1. We let $\operatorname{Unt}(w)$ be the untimed version of $w$ obtained by erasing all the durations 


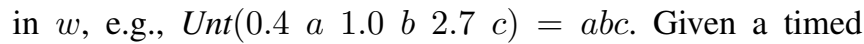
language $L$, we let $\operatorname{Unt}(L)=\{\operatorname{Unt}(w) \mid w \in L\}$.

Let $\pi_{/ \Sigma^{\prime}}$ be the projection of timed words of $T W(\Sigma)$ over timed words of $T W\left(\Sigma^{\prime}\right)$. When projecting a timed word $w$ on a sub-alphabet $\Sigma^{\prime} \subseteq \Sigma$, the durations elapsed between two events are set accordingly: for instance $\boldsymbol{\pi}_{/\{a, c\}}\left(\begin{array}{llllll}0.4 & a & 1.0 & b & 2.7 & c\end{array}\right)=0.4 a 3.7 c$ (projection erases some letters but keep the time elapsed between two letters). Given $\Sigma^{\prime} \subseteq \Sigma, \boldsymbol{\pi}_{/ \Sigma^{\prime}}(L)=\left\{\boldsymbol{\pi}_{/ \Sigma^{\prime}}(w) \mid w \in L\right\}$.

\section{Timed Automata}

Timed automata (TA) are finite automata extended with real-valued clocks to specify timing constraints between occurrences of events. For a detailed presentation of the fundamental results for timed automata, the reader is referred to the seminal paper of R. Alur and D. Dill [10].

Definition 1 (Timed Automaton): A Timed Automaton A is a tuple $\left(L, l_{0}, X, \Sigma_{\tau}, E, \operatorname{Inv}, F, R\right)$ where: $L$ is a finite set of locations; $l_{0}$ is the initial location; $X$ is a finite set of clocks; $\Sigma$ is a finite set of actions; $E \subseteq L \times \mathcal{C}(X) \times$ $\Sigma_{\tau} \times 2^{X} \times L$ is a finite set of transitions; for $\left(\ell, g, a, r, \ell^{\prime}\right) \in$ $E, g$ is the guard, a the action, and $r$ the reset set; Inv $\in$ $\mathcal{C}(X)^{L}$ associates with each location an invariant; as usual we require the invariants to be conjunctions of constraints of the form $x \preceq c$ with $\preceq \in\{<, \leq\} . F \subseteq L$ and $R \subseteq L$ are respectively the final and repeated sets of locations.

A state of $A$ is a pair $(\ell, v) \in L \times \mathbb{R}_{\geq 0}^{X}$. A run $\varrho$ of $A$ from $\left(\ell_{0}, v_{0}\right)$ is a (finite or infinite) sequence of alternating delay and discrete moves:

$$
\begin{aligned}
\varrho= & \left(\ell_{0}, v_{0}\right) \stackrel{\delta_{0}}{\longrightarrow}\left(\ell_{0}, v_{0}+\delta_{0}\right) \stackrel{a_{0}}{\longrightarrow}\left(\ell_{1}, v_{1}\right) \cdots \\
& \cdots \stackrel{a_{n-1}}{\longrightarrow}\left(\ell_{n}, v_{n}\right) \stackrel{\delta_{n}}{\longrightarrow}\left(\ell_{n}, v_{n}+\delta_{n}\right) \cdots
\end{aligned}
$$

s.t. for every $i \geq 0$ :

- $v_{i}+\delta=\operatorname{Inv}\left(\ell_{i}\right)$ for $0 \leq \delta \leq \delta_{i}$;

- there is some transition $\left(\ell_{i}, g_{i}, a_{i}, r_{i}, \ell_{i+1}\right) \in E$ s.t. : (i) $v_{i}+\delta_{i} \models g_{i}$ and $(i i) v_{i+1}=\left(v_{i}+\delta_{i}\right)\left[r_{i}\right]$.

The set of finite (resp. infinite) runs from a state $s$ is denoted Runs $^{*}(s, A)$ (resp. Runs ${ }^{\omega}(s, A)$ ) and we define Runs ${ }^{*}(A)=$ Runs $^{*}\left(\left(l_{0}, \mathbf{0}\right), A\right), \operatorname{Runs}^{\omega}(A)=$ Runs $^{\omega}\left(\left(l_{0}, \mathbf{0}\right), A\right)$ and finally $\operatorname{Runs}(A)=\operatorname{Runs}^{*}(A) \cup \operatorname{Runs}^{\omega}(A)$. If $\varrho$ is finite and ends in $s_{n}$, we let last $(\varrho)=s_{n}$. Because of the denseness of the time domain, the transition graph of $A$ is infinite (uncountable number of states and delay edges). The $\operatorname{trace}, \operatorname{tr}(\varrho)$, of a run $\varrho$ is the timed word $\pi_{/ \Sigma}\left(\delta_{0} a_{0} \delta_{1} a_{1} \cdots a_{n} \delta_{n} \cdots\right)$. We let $\operatorname{Dur}(\varrho)=\operatorname{Dur}(\operatorname{tr}(\varrho))$. For $V \subseteq \operatorname{Runs}(A)$, we let $\operatorname{Tr}(V)=$ $\{\operatorname{tr}(\varrho) \mid \varrho \in V\}$.

A finite (resp. infinite) timed word $w$ is accepted by $A$ if it is the trace of a run of $A$ that ends in an $F$-location (resp. a run that reaches infinitely often an $R$-location). $\mathcal{L}^{*}(A)$ (resp. $\mathcal{L}^{\omega}(A)$ ) is the set of traces of finite (resp. infinite) timed words accepted by $A$, and $\mathcal{L}(A)=\mathcal{L}^{*}(A) \cup \mathcal{L}^{\omega}(A)$ is the set of timed words accepted by $A$. In the sequel we often omit the sets $R$ and $F$ in TA and this implicitly means $F=L$ and $R=\varnothing$.

A timed automaton $A$ is deterministic if there is no $\tau$ labelled transition in $A$, and if, whenever $\left(\ell, g, a, r, \ell^{\prime}\right)$ and $\left(\ell, g^{\prime}, a, r^{\prime}, \ell^{\prime \prime}\right)$ are transitions of $A, g \wedge g^{\prime} \equiv$ FALSE. $A$ is complete if from each state $(\ell, v)$, and for each action $a$, there is a transition $\left(\ell, g, a, r, \ell^{\prime}\right)$ such that $v \models g$. We note DTA the class of deterministic timed automata.

\section{Region Graph of a TA}

The region graph $R G(A)$ of a TA $A$ is a finite quotient of the infinite graph of $A$ which is time-abstract bisimilar to $A$ [10]. It is a finite automaton (FA) on the alphabet $E^{\prime}=$ $E \cup\{\tau\}$. The states of $R G(A)$ are pairs $(\ell, r)$ where $\ell \in L$ is a location of $A$ and $r$ is a region of $\mathbb{R}_{>0}^{X}$. More generally, the edges of the graph are tuples $\left(s, t, s^{\prime}\right)$ where $s, s^{\prime}$ are states of $R G(A)$ and $t \in E^{\prime}$. Genuine unobservable moves of $A$ labelled $\tau$ are labelled by tuples of the form $\left(s,(g, \tau, r), s^{\prime}\right)$ in $R G(A)$. An edge $(g, \lambda, R)$ in the region graph corresponds to a discrete transition of $A$ with guard $g$, action $\lambda$ and reset set $R$. A $\tau$ move in $R G(A)$ stands for a delay move to the time-successor region. The initial state of $R G(A)$ is $\left(l_{0}, \mathbf{0}\right)$. A final (resp. repeated) state of $R G(A)$ is a state $(\ell, r)$ with $\ell \in F$ (resp. $\ell \in R$ ). A fundamental property of the region graph [10] is:

Theorem 1 ([10]): $\mathcal{L}(R G(A))=\operatorname{Unt}(\mathcal{L}(A))$.

The (maximum) size of the region graph is exponential in the number of clocks and in the maximum constant of the automaton $A$ (see [10]): $|R G(A)|=|L| \cdot|X| ! \cdot 2^{|X|} \cdot K^{|X|}$ where $K$ is the largest constant used in $A$.

\section{E. Product of TA}

Definition 2 (Product of two TA): Let $A_{i}=\left(L_{i}, l_{0}^{i}, X_{i}\right.$, $\left.\Sigma_{\tau}^{i}, E_{i}, \operatorname{Inv}_{i}\right)$ for $i \in\{1,2\}$, be two TA s.t. $X_{1} \cap X_{2}=\varnothing$. The product of $A_{1}$ and $A_{2}$ is the TA $A_{1} \times A_{2}=\left(L, l_{0}, X, \Sigma_{\tau}\right.$, $E$, Inv) given by: $L=L_{1} \times L_{2} ; l_{0}=\left(l_{0}^{1}, l_{0}^{2}\right) ; \Sigma=\Sigma^{1} \cup \Sigma^{2}$; $X=X_{1} \cup X_{2}$; and $E \subseteq L \times \mathcal{C}(X) \times \Sigma_{\tau} \times 2^{X} \times L$ and $\left(\left(\ell_{1}, \ell_{2}\right), g_{1,2}, \sigma, r,\left(\ell_{1}^{\prime}, \ell_{2}^{\prime}\right)\right) \in E$ if:

- either $\sigma \in\left(\Sigma_{1} \cap \Sigma_{2}\right) \backslash\{\tau\}$, and $(i)\left(\ell_{k}, g_{k}, \sigma, r_{k}, \ell_{k}^{\prime}\right) \in$ $E_{k}$ for $k=1$ and $k=2$; (ii) $g_{1,2}=g_{1} \wedge g_{2}$ and (iii) $r=r_{1} \cup r_{2}$

- or for $k=1$ or $k=2, \sigma \in\left(\Sigma_{k} \backslash \Sigma_{3-k}\right) \cup\{\tau\}$, and (i) $\left(\ell_{k}, g_{k}, \sigma, r_{k}, \ell_{k}^{\prime}\right) \in E_{k} ;(i i) g_{1,2}=g_{k}$ and (iii) $r=r_{k}$; and finally $\operatorname{Inv}\left(\ell_{1}, \ell_{2}\right)=\operatorname{Inv}\left(\ell_{1}\right) \wedge \operatorname{Inv}\left(\ell_{2}\right)$.

\section{Fault Diagnosis Problems \& Known Results}

\section{A. The Model}

To model timed systems with faults, we use timed automata on the alphabet $\Sigma_{\tau, f}=\Sigma_{\tau} \cup\{f\}$ where $f$ is the faulty (and unobservable) event. We only consider one type of fault, but the results we give are valid for many types of faults $\left\{f_{1}, f_{2}, \cdots, f_{n}\right\}$ : indeed solving the many types diagnosability problem amounts to solving $n$ one type diagnosability problems [7]. The observable events are given by $\Sigma_{o} \subseteq \Sigma$ and $\tau$ is always unobservable.

The system we want to supervise is given by a TA $A=$ $\left(L, l_{0}, X, \Sigma_{\tau, f}, E\right.$, Inv $)$. Fig. 1 gives an example of such a system. Invariants in the automaton $\mathcal{A}$ are written within square brackets as in $[x \leq 3]$. 


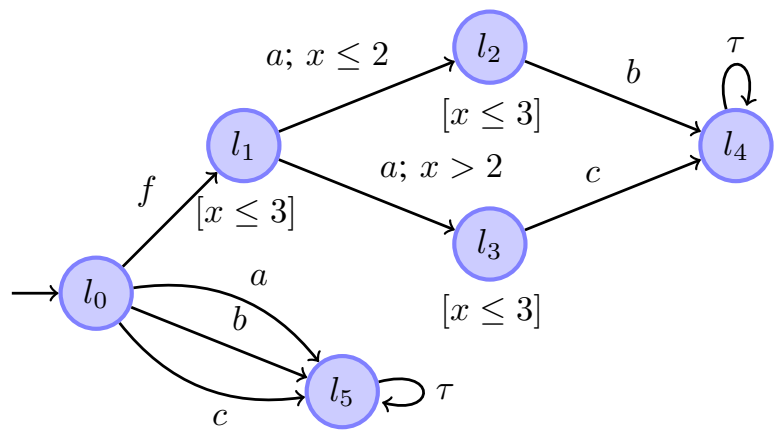

Figure 1. The Timed Automaton $\mathcal{A}$

Let $\Delta \in \mathbb{N}$. A run of $A$

$$
\begin{aligned}
\varrho= & \left(\ell_{0}, v_{0}\right) \stackrel{\delta_{0}}{\longrightarrow}\left(\ell_{0}, v_{0}+\delta_{0}\right) \stackrel{a_{0}}{\longrightarrow}\left(\ell_{1}, v_{1}\right) \cdots \\
& \cdots \stackrel{a_{n-1}}{\longrightarrow}\left(\ell_{n}, v_{n}\right) \stackrel{\delta_{n}}{\longrightarrow}\left(\ell_{n}, v_{n}+\delta\right) \cdots
\end{aligned}
$$

is $\Delta$-faulty if: (1) there is an index $i$ s.t. $a_{i}=f$ and (2) the duration of the run $\varrho^{\prime}=\left(\ell_{i}, v_{i}\right) \stackrel{\delta_{i}}{\longrightarrow} \cdots \stackrel{\delta_{n}}{\longrightarrow}\left(\ell_{n}, v_{n}+\right.$ $\left.\delta_{n}\right) \cdots$ is larger than $\Delta$. We let Faulty ${ }_{\geq \Delta}(A)$ be the set of $\Delta$-faulty runs of $A$. Note that by definition, if $\Delta^{\prime} \geq \Delta$ then Faulty $_{\geq \Delta^{\prime}}(A) \subseteq$ Faulty $_{\geq \Delta}(A)$. We let Faulty $(A)=$ $\cup_{\Delta \geq 0}$ Faulty $_{\geq \Delta}(A)=$ Faulty $_{\geq 0}(A)$ be the set of faulty runs of $\bar{A}$, and $\operatorname{NonFaulty}(A)=\bar{R} u n s(A) \backslash \operatorname{Faulty}(A)$ be the set of non-faulty runs of $A$. Moreover we use

$$
\text { Faulty }_{\geq \Delta}^{\text {tr }}(A)=\operatorname{Tr}\left(\text { Faulty }_{\geq \Delta}(A)\right)
$$

and

$$
\operatorname{NonFaulty}^{\text {tr }}(A)=\operatorname{Tr}(\operatorname{NonFaulty}(A))
$$

which are the traces $\square^{1}$ of $\Delta$-faulty and non-faulty runs of $A$.

\section{B. Diagnosers}

The purpose of fault diagnosis is to detect a fault as soon as possible. Faults are unobservable and only the events in $\Sigma_{o}$ can be observed as well as the time elapsed between these events. Whenever the system generates a timed word $w$, the observer can only see $\boldsymbol{\pi}_{/ \Sigma_{o}}(w)$. If an observer can detect faults in this way it is called a diagnoser. A diagnoser must detect a fault within a given delay $\Delta \in \mathbb{N}$.

Definition $3\left(\left(\Sigma_{o}, \Delta\right)\right.$-Diagnoser $)$ : Let $A$ be a TA over the alphabet $\Sigma_{\tau, f}, \Sigma_{o} \subseteq \Sigma$ and $\Delta \in \mathbb{N}$. A $\left(\Sigma_{o}, \Delta\right)$-diagnoser for $A$ is a mapping $D: T W^{*}\left(\Sigma_{o}\right) \rightarrow\{0,1\}$ such that:

- for each $\varrho \in \operatorname{NonFaulty}(A), D\left(\pi_{/ \Sigma_{o}}(\varrho)\right)=0$,

- for each $\varrho \in$ Faulty $_{\geq \Delta}(A), D\left(\boldsymbol{\pi}_{/ \Sigma_{o}}(\varrho)\right)=1$.

$A$ is $\left(\Sigma_{o}, \Delta\right)$-diagnosable if there exists a $\left(\Sigma_{o}, \Delta\right)$-diagnoser for $A$. $A$ is $\Sigma_{o}$-diagnosable if there is some $\Delta \in \mathbb{N}$ s.t. $A$ is $\left(\Sigma_{o}, \Delta\right)$-diagnosable.

Example 1: The TA $\mathcal{A}$ in Fig. 1 with $\Sigma=\Sigma_{o}=\{a, b, c\}$ is $(\Sigma, 3)$-diagnosable. For the timed words with an $a$ followed by either a $b$ or a $c$ a fault must have occurred.

\footnotetext{
${ }^{1}$ Notice that $\operatorname{tr}(\varrho)$ erases $\tau$ and $f$.
}

Otherwise no fault should be reported. If $\Sigma_{o}=\{b\}$, in $\mathcal{A}$ there are two runs:

$\rho_{1}=\left(l_{0}, 0\right) \stackrel{f}{\longrightarrow}\left(l_{1}, 0\right) \stackrel{a}{\longrightarrow}\left(l_{2}, 0\right) \stackrel{3}{\longrightarrow}\left(l_{2}, 3\right) \stackrel{b}{\longrightarrow}\left(l_{4}, 3\right) \cdots$

$\rho_{2}=\left(l_{0}, 0\right) \stackrel{3}{\rightarrow}\left(l_{0}, 3\right) \stackrel{b}{\longrightarrow}\left(l_{5}, 3\right) \cdots$

that satisfy $\operatorname{tr}\left(\rho_{1}\right)=\operatorname{tr}\left(\rho_{2}\right)$, and thus $\mathcal{A}$ is not $(\{b\}, 3)$-diagnosable. To diagnose a fault in $\mathcal{A}$, a must be observed.

\section{Classical Diagnosis Problems}

Assume $A=\left(L, \ell_{0}, X, \Sigma_{\tau, f}, E, I n v\right)$ is a TA . The classical fault diagnosis problems are the following:

Problem 1 (Bounded or $\Delta$-Diagnosability):

INPUTS: A TA $A, \Sigma_{o} \subseteq \Sigma$, and $\Delta \in \mathbb{N}$.

PRoBlem: Is $A\left(\Sigma_{o}, \Delta\right)$-diagnosable?

Problem 2 (Diagnosability):

INPUTS: A TA $A$ and $\Sigma_{o} \subseteq \Sigma$.

PROBlem: Is $A \Sigma_{o}$-diagnosable?

Problem 3 (Maximum delay):

INPUTS: A TA $A$ and and $\Sigma_{o} \subseteq \Sigma$.

Problem: If $A$ is $\Sigma_{o}$-diagnosable, what is the minimum $\Delta$ s.t. $A$ is $\left(\Sigma_{o}, \Delta\right)$-diagnosable ?

According to Definition $3, A$ is $\Sigma_{o}$-diagnosable, iff, there is some $\Delta \in \mathbb{N}$ s.t. $A$ is $\left(\Sigma_{o}, \Delta\right)$-diagnosable. Thus $A$ is not $\Sigma_{o}$-diagnosable iff $\forall \Delta \in \mathbb{N}, A$ is not $\left(\Sigma_{o}, \Delta\right)$-diagnosable. Moreover a trace based definition of $\left(\Sigma_{o}, \Delta\right)$-diagnosability can be stated as $A$ is $\left(\Sigma_{o}, \Delta\right)$-diagnosable iff

$$
\boldsymbol{\pi}_{/ \Sigma_{o}}\left(\text { Faulty }_{\geq \Delta}^{t r}(A)\right) \cap \boldsymbol{\pi}_{/ \Sigma_{o}}\left(\text { NonFaulty }^{t r}(A)\right)=\varnothing .
$$

This gives a necessary and sufficient condition for non $\Sigma_{O^{-}}$ diagnosability:

$A$ is not $\Sigma_{o}$-diagnosable $\Longleftrightarrow\left\{\begin{array}{c}\forall \Delta \in \mathbb{N}, \\ \exists \rho \in \operatorname{NonFaulty}(A) \\ \exists \rho^{\prime} \in \text { Faulty }_{\geq \Delta}(A) \text { s.t. } \\ \pi_{/ \Sigma_{o}}(\rho)=\pi_{/ \Sigma_{o}}\left(\rho^{\prime}\right),\end{array}\right.$

or in other words, there is no pair of runs $\left(\rho_{1}, \rho_{2}\right)$ with $\rho_{1} \in$ Faulty $_{\geq \Delta}(A), \rho_{2} \in \operatorname{NonFaulty}(A)$ the $\Sigma_{o}$-traces of which are equal.

Complexity results for the diagnosis problems on timed automata were established in [8] (see [11] for a comprehensive study) and Problems 1,3 are PSPACE-complete (note that PSPACE-completeness already holds for $\Sigma_{o}=\Sigma$ ).

\section{Sensor Minimization with Static Observers}

In this section, we extend the results of [1] to systems given by TA.

Problem 4 (Minimum Cardinality Set):

INPUTS: A TA $A=\left(L, \ell_{0}, X, \Sigma_{\tau, f}, E\right.$, Inv $)$ and $n \in \mathbb{N}$. PROBLEM:

(A) Is there any set $\Sigma_{o} \subseteq \Sigma$, with $\left|\Sigma_{o}\right|=n$ s.t. $A$ is $\Sigma_{o^{-}}$ diagnosable?

(B) If the answer to (A) is "yes", compute the minimum value for $n$.

\footnotetext{
${ }^{2}$ This definition does not take into account Zeno runs; this is not difficult to add and the reader is referred to [11] for more details.
} 
Theorem 2: Problem 4 is PSPACE-complete.

Proof: PSPACE-easiness for (A) can be established as follows: guess a set $\Sigma_{o}$ with $\left|\Sigma_{o}\right|=n$ and check (in PSPACE) whether $A$ is $\Sigma_{o}$-diagnosable. This proves NPSPACE and thus in PSPACE. PSPACE-hardness follows from the reduction of Problem 2 to Problem 4 (A) with $n=|\Sigma|$. This establishes PSPACE-completeness for (A). Computing the minimum $n$ can be done using a binary search (dichotomy) and thus (B) is also in PSPACE.

The previous results also hold in a more general setting using masks. Masks are useful to capture the notion of distinguishability among observable events. Indeed, there are cases where two events $a$ and $b$ are observable but not distinguishable, that is, the diagnoser knows that $a$ or $b$ occurred, but not which of the two. This is not the same as considering $a$ and $b$ to be unobservable, since in that case the diagnoser would not be able to detect the occurrence of $a$ or $b$. Distinguishability of events is captured by the notion of a mask [12].

Definition 4 (Mask): A mask $(M, n)$ (of size $n$ ) over $\Sigma$ is a total, surjective function $M: \Sigma \rightarrow\{\mathbf{1}, \cdots, \mathbf{n}\} \cup\{\varepsilon\}$. $M$ induces a morphism $M^{*}: T W^{*}(\Sigma) \rightarrow T W^{*}(\{\mathbf{1}, \cdots, \mathbf{n}\})$, where $M^{*}(\varepsilon)=\varepsilon$ and $M^{*}(a . \rho)=M(a) \cdot M^{*}(\rho)$, for $a \in \Sigma$ and $\rho \in \Sigma^{*}$. For example, if $\Sigma=\{a, b, c, d\}, n=2$ and $M(a)=M(d)=\mathbf{1}, M(c)=\mathbf{2}, M(b)=\varepsilon$, then we have $\left.M^{*}(a) 0.4 b 0.2 c 1.1 \quad b \quad 0.7 d\right)=10.621 .81$.

Definition $5((M, n), \Delta)$-diagnoser $)$ : Let $(M, n)$ be a mask over $\Sigma$. A mapping $D: T W^{*}(\{\mathbf{1}, \cdots, \mathbf{n}\}) \rightarrow\{0,1\}$ is a $((M, n), \Delta)$-diagnoser for $A$ if:

- for each $\rho \in \operatorname{NonFaulty}(A), D\left(M^{*}(\operatorname{tr}(\rho))\right)=0$;

- for each $\rho \in$ Faulty $_{\geq k}(A), D\left(M^{*}(\operatorname{tr}(\rho))\right)=1$.

$A$ is $((M, n), \Delta)$-diagnosable if there is a $((M, n), \Delta)$ diagnoser for $A$. $A$ is said to be $(M, n)$-diagnosable if there is some $\Delta$ such that $A$ is $((M, n), \Delta)$-diagnosable. Given a mask $(M, n)$ and $A$, checking whether $A$ is $(M, n)$ diagnosable can be done in PSPACE: it suffices to replace each event $a \in \Sigma$ by $M(a)$ and check for diagnosability. It is PSPACE-complete as using an identity mask of cardinality $|\Sigma|$ solves Problem 2

The counterpart of Problem 4 with masks is the following: Problem 5 (Minimum Cardinality Mask):

INPUTS: A TA $A=\left(L, \ell_{0}, X, \Sigma_{\tau, f}, E, \operatorname{Inv}\right)$ and $n \in \mathbb{N}$. PROBLEM:

(A) Is there any mask $(M, n)$, s.t. $A$ is $(M, n)$-diagnosable?

(B) If the answer to (A) is "yes", compute the minimum value for $n$.

Theorem 3: Problem 5 is PSPACE-complete.

Proof: PSPACE-easiness is proved by: 1) guessing a mask $(M, n)$ and checking (in PSPACE) that $A$ is $(M, n)$ diagnosable. PSPACE-hardness is proved as follows. If there is a mask $(M, n)$ with $n=|\Sigma|$ s.t. $A$ is $(M, n)$-diagnosable, then, as $M$ is surjective, it must be the case that $M$ is a oneto-one mapping from $\Sigma$ to $\{\mathbf{1}, \cdots, \mathbf{n}\}$. It follows that $A$ is $\Sigma$ diagnosable. Conversely, assume $\Sigma=\left\{a_{1}, \cdots, a_{n}\right\}$. If $A$ is $\Sigma$-diagnosable then there is a mask $(M,|\Sigma|)$ with $M\left(a_{i}\right)=$ $i$ s.t. $A$ is $(M,|\Sigma|)$-diagnosable. Hence Problem $5(\mathrm{~A})$ is
PSPACE-complete. Problem 5. (B) can be solved in PSPACE as well using a binary search. It is not difficult to reduce reachability for TA with one action to checking whether there is a mask of size 1 and thus Problem 5.(B) is PSPACEcomplete.

Remark 1: The assumption that a mask is surjective can be lifted still preserving Theorem 3 . Indeed, if there is a mask $(M,|\Sigma|)$ s.t. $A$ is $(M,|\Sigma|)$-diagnosable and $M$ is not surjective, then we can build $\left(M^{\prime},|\Sigma|\right)$ with $M^{\prime}$ surjective s.t. $A$ is $\left(M^{\prime},|\Sigma|\right)$-diagnosable (intuitively, $M^{\prime}$ is more discriminating than $M$ and has a greater distinguishing power).

\section{Sensor Minimization with Dynamic Observers}

The use of dynamic observers was already advocated for DES in [1], [3]. We start with an example that shows that dynamically choosing what to observe can be even more efficient using timing information.

Example 2: Let $\mathcal{A}$ be the automaton of Figure 1. To diagnose $\mathcal{A}$, we can use a dynamic observer that switches $a$, $b$ and $c$-sensors on/off. If we do not measure time, to be able to detect faults in $\mathcal{A}$, we have to switch the $a$ sensor on at the beginning. When an $a$ has occurred, we must be ready for either an $b$ or a $c$ and therefore, switch on the $b$ and $c$ sensors on. A dynamic observer must thus first observe $\{a\}$ and after an occurrence of $a$, observe $\{b, c\}$.

If the observer can measure time using a clock, say $y$, it can first switch the $a$ sensor on. If an $a$ occurs when $y \leq 2$, then switch the $b$ sensor on and if $y>2$ switch the $c$ sensor on. This way the observer never has to observe more than event at each point in time.

\section{A. Dynamic Observers}

The choice of the events to observe can depend on the choices the observer has made before and on the observations (event, time-stamp) it has made. Moreover an observer may have unbounded memory. The following definition extends the notion of observers introduced in [1] to the timed setting.

Definition 6 (Observer): An observer Obs over $\Sigma$ is a deterministic and complete timed automaton $O b s=\left(N, n_{0}, Y\right.$, $\Sigma, \delta$, Inv $\left._{\text {TRUE }}\right)$ together with a mapping $O: N \rightarrow 2^{\Sigma}$, where $N$ is a (possibly infinite) set of locations, $n_{0} \in N$ is the initial location, $\Sigma$ is the set of observable events, $\delta: N \times \Sigma \times \mathcal{C}(Y) \rightarrow N \times 2^{Y}$ is the transition function (a total function), and $O$ is a labeling function that specifies the set of events that the observer wishes to observe when it is at location $n$. The invarian $3{ }^{3} v_{\text {TRUE }}$ maps every location to TRUE, implying that an observer cannot prevent time from elapsing. We require that, for any location $n$ and any $a \in \Sigma$, if $a \notin O(n)$ then $\delta(n, a, \cdot)=(n, \varnothing)$ : this means the observer does not change its location nor resets its clocks when an event it has chosen not to observe occurs.

As an observer is deterministic we let $\delta\left(n_{0}, w\right)$ denote the state $(n, v)$ reached after reading the timed word $w$ and $O\left(\delta\left(n_{0}, w\right)\right)$ is the set of events Obs observes after $w$.

\footnotetext{
${ }^{3}$ In the sequel, we omit the invariant when a TA is an observer, and replace it by the mapping $O$.
} 
An observer defines a transducer which is a mapping $\llbracket O b s \rrbracket$ : $T W^{*}(\Sigma) \rightarrow T W^{*}(\Sigma)$. Given a word $w, \llbracket O b s \rrbracket(w)$ is the output of the transducer on $w$. It is called the observation of $w$ by the observer $O b s$.

\section{B. Diagnosability with Dynamic Observers}

Definition 7 ( $($ Obs, $\Delta)$-diagnoser $)$ : Let $A$ be a TA over $\Sigma_{\tau, f}$ and $O b s$ be an observer over $\Sigma . D: T W^{*}(\Sigma) \rightarrow\{0,1\}$ is an $(O b s, \Delta)$-diagnoser for $A$ if:

- $\forall \rho \in \operatorname{NonFaulty}(A), D(\llbracket O b s \rrbracket(\operatorname{tr}(\rho)))=0$ and

- $\forall \rho \in$ Faulty $_{\geq \Delta}(A), D(\llbracket O b s \rrbracket(\operatorname{tr}(\rho)))=1$.

$A$ is $(O b s, \Delta)$-diagnosable if there is an $(O b s, \Delta)$-diagnoser for $A$. $A$ is $O b s$-diagnosable if there is some $\Delta$ such that $A$ is $(\mathrm{Obs}, \Delta)$-diagnosable.

We now show how to check Obs-diagnosability when the observer $\mathrm{Obs}$ is a DTA.

Problem 6 (Deterministic Timed Automata Observers): INPUTS: A TA $A=\left(L, \ell_{0}, X, \Sigma_{\tau, f}, E, I n v\right)$ and an observer given by a DTA $O b s=\left(N, n_{0}, Y, \Sigma, \delta, O\right)$.

\section{PROBLEM:}

(A) Is $A O b s$-diagnosable?

(B) If the answer to (A) is "yes", compute the minimum $\Delta \in \mathbb{N}$ s.t. $A$ is $(\mathrm{Obs}, \Delta)$-diagnosable.

Theorem 4: Problem 6 is PSPACE-complete.

Proof: PSPACE-hardness follows from the fact that taking an observer which always observes $\Sigma_{o} \subseteq \Sigma$ solves Problem 2. We prove that Problem 6 is in PSPACE. The following construction is an extension of the one for DES [3]. Recall that $O b s$ is complete. Define the timed automaton $A \otimes O b s=\left(L \times N,\left(\ell_{0}, n_{0}\right), X \cup Y, \Sigma_{\tau, f}, \rightarrow, \operatorname{In} v_{\otimes}\right)$ as follows: $\operatorname{In} v_{\otimes}(\ell, n)=\operatorname{Inv}(\ell)$ and the transition relation $\rightarrow$ is given by:

- $(\ell, n) \stackrel{\left(g \wedge g^{\prime}, \beta, R \cup Y^{\prime}\right)}{\longrightarrow}\left(\ell^{\prime}, n^{\prime}\right)$ iff $\exists \lambda \in \Sigma$ s.t. $\ell \stackrel{(g, \lambda, R)}{\longrightarrow}$ $\ell^{\prime},\left(n^{\prime}, Y^{\prime}\right)=\delta\left(n, \lambda, g^{\prime}\right)$ and $\beta=\lambda$ if $\lambda \in O(n), \beta=\tau$ otherwise;

- $(\ell, n) \stackrel{(g, \lambda, R)}{\longrightarrow}\left(\ell^{\prime}, n\right)$ iff $\exists \lambda \in\{\tau, f\}$ s.t. $\ell \stackrel{(g, \lambda, R)}{\longrightarrow} \ell^{\prime}$. The TA $A \otimes O b s$ is an unfolding of $A$ which reveals what is observable at each product location.

From the previous construction, it follows that: for each $\Delta \in \mathbb{N}, A$ is $(O b s, \Delta)$-diagnosable iff $A \otimes O b s$ is $(\Sigma, \Delta)$ diagnosable. As the size of $A \otimes O b s$ is $|A| \times|O b s|$, we can solve Problem 6(A) in PSPACE. Problem 6.(B) can also be solved using a binary search, in PSPACE.

\section{Synthesis of the Most Permissive Dynamic Diagnoser}

In this section we address the problem of synthesizing a DTA dynamic observer which ensures diagnosability. Following [3], we want to compute a most permissive observer ( $\varnothing$ if none exists), which gives a representation of all the good observers. Indeed, checking whether there exists a DTA observer $O b s$ s.t. $A$ is $O b s$-diagnosable is not an interesting problem: it suffices to check that $A$ is $\Sigma$-diagnosable as the DTA observer which observes $\Sigma$ continuously will be a solution.

When synthesizing (deterministic) timed automata, an important issue is the amount of resources the timed automaton can use: this can be formally defined [13] by the (number of) clocks, $Z$, that the automaton can use, the maximal constant max, and a granularity $\frac{1}{m}$. As an example, a TA of resource $\mu=\left(\{c, d\}, 2, \frac{1}{3}\right)$ can use two clocks, $c$ and $d$, and the clocks constraints using the rationals $-2 \leq k / m \leq 2$ where $k \in \mathbb{Z}$ and $m=3$. A resource $\mu$ is thus a triple $\mu=\left(Z, \max , \frac{1}{m}\right)$ where $Z$ is finite set of clocks, $\max \in \mathbb{N}$ and $\frac{1}{m} \in \mathbb{Q}>0$ is the granularity. DTA $\mu$ is the class of DTA of resource $\mu$.

Remark 2: Notice that the number of locations of the DTA in DTA $_{\mu}$ is not bounded and hence this family has an infinite (yet countable) number of elements.

We now focus on the following problem :

Problem 7 (Most Permissive Dynamic $\Delta$-Diagnoser):

InPUTS: A TA $A=\left(L, \ell_{0}, X, \Sigma_{\tau, f}, E, \operatorname{Inv}\right), \Delta \in \mathbb{N}$, and a resource $\mu=\left(Z, \max , \frac{1}{m}\right)$.

Problem: Compute the set $O$ of all observers in $\mathrm{DTA}_{\mu}$, s.t. $A$ is $(O b s, \Delta)$-diagnosable iff $O b s \in O$.

For DES, the previous problem can be solved by computing a most permissive observer, and we refer to [3] section 5.5 for the formal definition of the most permissive observer. This can be done in 2EXPTIME [3], and the solution is a reduction to a safety control problem under partial observation. For the timed case, we cannot use the same solution as controller synthesis under partial observation is undecidable [13]. The solution we present for Problem 7 is a modification of an algorithm originally introduced in [9].

\section{Fault Diagnosis with DTA [9]}

In case a TA $A$ is $\Sigma_{o}$-diagnosable, the diagnoser is a mapping [8] which performs a state estimate of $A$ after a timed word $w$ is read by $A$. For DES, it is obtained by determinizing the system, but we cannot always determinize a TA $A$ (see [10]). And unfortunately testing whether a timed automaton is determinizable is undecidable [14], [15].

P. Bouyer and F. Chevalier in [9] considers the problem of deciding whether there exists a diagnoser which is a DTA using resources in $\mu$ :

Problem $8\left(\right.$ DTA $_{\mu} \Delta$-Diagnoser [9]):

InPUTS: A TA $A=\left(L, \ell_{0}, X, \Sigma_{\tau, f}, E, \operatorname{Inv}\right), \Delta \in \mathbb{N}$, and a resource $\mu=\left(Z, \max , \frac{1}{m}\right)$.

Problem: Is there any $D \in \mathrm{DTA}_{\mu}$ s.t. $A$ is $(D, \Delta)$-diagnosable ?

Theorem 5 ([9]): Problem 8 is 2EXPTIME-complete.

The solution to the previous problem is based on the construction of a two-player game, the solution of which gives the set of all $\mathrm{DTA}_{\mu}$ diagnosers (the most permissive diagnosers) which can diagnose $A$ (or $\varnothing$ is there is none).

We recall here the construction of the two-player game.

Let $A=\left(L, \ell_{0}, X, \Sigma_{\tau, f}, \rightarrow\right.$, Inv $)$ be a TA, $\Sigma_{o} \subseteq \Sigma$. Define $A(\Delta)=\left(L_{1} \cup L_{2} \cup L_{3}, \ell_{0}^{1}, X \cup\{z\}, \Sigma_{\tau, f}, \rightarrow_{\Delta}\right.$, Inv $\left._{\Delta}\right)$ as follows:

- $L_{i}=\left\{\ell^{i}, \ell \in L\right\}$, for $i \in\{1,2,3\}$, i.e., $L_{i}$ elements are copies of the locations in $L$,

- $z$ is (new) clock not in $X$,

- for $\ell \in L, \operatorname{Inv}\left(\ell^{1}\right)=\operatorname{Inv}(\ell), \operatorname{Inv}\left(\ell^{2}\right)=\operatorname{Inv}(\ell) \wedge z \leq \Delta$, and $\operatorname{Inv}\left(\ell^{3}\right)=$ TRUE,

- the transition relation is given by: 
- for $i \in\{1,2,3\}, \ell^{i} \stackrel{(g, a, R)}{\longrightarrow} \Delta \ell^{\prime i}$ if $a \neq f$ and $\ell \stackrel{(g, a, R)}{\longrightarrow} \ell^{\prime}$,

- for $i \in\{2,3\}, \ell^{i} \stackrel{(g, f, R)}{\longrightarrow} \Delta \ell^{i}$ if $a \neq f$ and $\ell \stackrel{(g, f, R)}{\longrightarrow} \ell^{\prime}$,

- $\ell^{1} \stackrel{(g, f, R \cup\{z\})}{\longrightarrow} \Delta \ell^{\prime 2}$ if $a \neq f$ and $\ell \stackrel{(g, f, R)}{\longrightarrow} \ell^{\prime}$, - $\ell^{2} \stackrel{(z=\Delta, \tau, \varnothing)}{\longrightarrow} \Delta \ell^{3}$.

The previous construction creates 3 copies of $A$ : the system starts in copy 1 , when a fault occurs it switches to copy 2 , resetting the clock $z$, and when in copy 2 (a fault has occurred) it can switch to copy 3 after $\Delta$ time units. We can then define $L_{1}$ as the non-faulty locations, and $L_{3}$ as the $\Delta$-faulty locations.

Given a resource $\mu=\left(Y, \max , \frac{1}{m}\right)(X \cap Y=\varnothing)$, a minimal guard for $\mu$ is a guard which defines a region of granularity $\mu$. We define the (symbolic) universal automaton $\mathcal{U}=\left(\{0\},\{0\}, Y, \Sigma, E_{\mu}, I n v_{\mu}\right)$ by:

- $\operatorname{In} v_{\mu}(0)=$ TRUE,

- $(0, g, a, R, 0) \in E_{\mu}$ for each $(g, a, R)$ s.t. $a \in \Sigma, R \subseteq$ $Y$, and $g$ is a minimal guard for $\mu$.

$\mathcal{U}$ is finite because $E_{\mu}$ is finite. Nevertheless $\mathcal{U}$ is not deterministic because it can choose to reset different sets of clocks $Y$ for a pair "(guard, letter)" $(g, a)$. To diagnose $A$, we have to find when a set of clocks has to be reset. This can provide enough information to distinguish $\Delta$-faulty words from non-faulty words.

The algorithm of [9] requires the following steps:

1) define the region graph $R G(A(\Delta) \times \mathcal{U})$,

2) compute a projection of this region graph:

- let $(g, a, R)$ be a label of an edge in $R G(A(\Delta) \times \mathcal{U})$,

- let $g^{\prime}$ be the unique minimal guard s.t. $\llbracket g \rrbracket \subseteq \llbracket g^{\prime} \rrbracket$;

- define the projection $p_{\mathcal{U}}(g, a, R)$ by $\left(g^{\prime}, \lambda, R \cap Y\right)$ with $\lambda=a$ if $a \in \Sigma_{o}$ and $p_{\mathcal{U}}(g, a, R)=\tau$ otherwise.

The projected automaton $p_{\mathcal{U}}(R G(A(\Delta) \times \mathcal{U}))$ is the automaton $R G(A(\Delta) \times \mathcal{U})$ where each label $\alpha$ is replaced by $p_{\mathcal{U}}(\alpha)$.

3) determinize $p_{\mathcal{U}}(R G(A(\Delta) \times \mathcal{U})$ ) (removing $\tau$ actions) and obtain $H_{A, \Delta, \mu}$,

4) build a two-player safety game $G_{A, \Delta, \mu}$ as follows:

- each transition $s \stackrel{(g, a, Y)}{\longrightarrow} s^{\prime}$ in $H_{A, \Delta, \mu}$ yields a transition in $G_{A, \Delta, \mu}$ of the form:

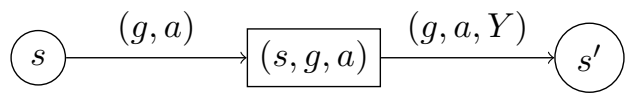

- the round-shaped state are the states of Player 1, whereas the square-shaped states are Player 0 states (the choice of the clocks to reset).

- the Bad states (for Player 0) are the states of the form $\left\{\left(\ell_{1}, r_{1}\right),\left(\ell_{2}, r_{2}\right), \cdots,\left(\ell_{k}, r_{k}\right)\right\}$ with both a $\Delta$-faulty (in $L_{3}$ ) and a non-faulty (in $L_{1}$ ) location.

The main results of [9] are:

- there is a TA $D \in \mathrm{DTA}_{\mu}$ s.t. $A$ is $(D, \Delta)$-diagnosable iff Player 0 can win the safety game "avoid Bad" $G_{A, \Delta, \mu}$,

- it follows that Problem 8 can be solved in 2EXPTIME as $G_{A, \Delta, \mu}$ has size doubly exponential in $A, \Delta$ and $\mu$,
- the acceptance problem for Alternating Turing machines of exponential space can be reduced to Problem 8 and thus it is 2EXPTIME-hard.

\section{E. Problem 7 is in 2EXPTIME}

We now show how to modify the previous algorithm to solve Problem 7, and obtain the following result:

Theorem 6: Problem 7 can be solved in 2EXPTIME. Proof: We modify the previous algorithm as follows:

1) the automaton $\mathcal{U}$ is defined as follows: each location corresponds to a choice of a subset of events to observe. Define the (symbolic) universal automaton $\mathcal{U}^{\prime}=$ $\left(2^{\Sigma}, 2^{\Sigma}, Y, \Sigma, E_{\mu}, \operatorname{In} v_{\mu}\right)$ by:

- for $S \in 2^{\Sigma}, \operatorname{Inv}_{\mu}(S)=$ TRUE,

- $\left(S, g, a, R, S^{\prime}\right) \in E_{\mu}$ for each $S, S^{\prime} \in 2^{\Sigma},(g, a, R)$ s.t. $a \in \Sigma, R \subseteq Y$, and $g$ is a minimal guard for $\mu$.

2) when computing $R G\left(A(\Delta) \times \mathcal{U}^{\prime}\right)$ ), the set of observable events (step 2 in the algorithm of section V-D) are defined according to the location $S$ of $\mathcal{U}^{\prime}$. Formally, the projection of $a \in \Sigma$ is $a$ if the location of $\mathcal{U}^{\prime}$ is $S$ and $a \in S$ and $\tau$ otherwise.

The size of $\left.R G\left(A(\Delta) \times \mathcal{U}^{\prime}\right)\right)$ is $|L| \cdot 2^{|\Sigma|} \cdot|X \cup Y| ! \cdot K^{|X \cup Y|}$ where $K$ is the maximal constant of $A \times \mathcal{U}^{\prime}$; it is thus exponential in $\mu$ and $\Sigma$. The determinization is thus doubly exponential in $A, \mu$ and $\Sigma$. We can then build a new game $G_{A, \Delta, \mu}^{\prime}$ as described in section V-D before. The proof that the most permissive strategy in the new game $G_{A, \Delta, \mu}^{\prime}$ is the most permissive observer is along the lines of the one given in [9] with minor modifications. Solving a safety game is linear in the size of the game and thus computing the most permissive observer of resource $\mu$ can de done in 2EXPTIME.

Remark 3: In [9] it is also proved that for Event Recording Automata (ERA) [16] Problem 8 becomes PSPACEcomplete. This result does not carry over in our case, as there is still an exponential step with the choice of the sets of events to be observed.

\section{OPTIMAL DYNAMIC OBSERVERS}

In this section we extend the notion of cost defined for finite state observers in [3] to the case of timed observers.

\section{A. Weighted/Priced Timed Automata}

Weighted/priced timed automata were introduced in [17], [18] and they extend TA with prices/costs/weights on the time elapsing and discrete transitions.

Definition 8 (Priced Timed Automata): A priced timed automaton (PTA) is a pair $(A$, Cost $)$ where $A=\left(L, \ell_{0}, X\right.$, $\left.\Sigma_{\tau, f}, E, I n v\right)$ is a timed automaton and Cost is a cost function which is a mapping from $L \cup E$ to $\mathbb{N}$.

Let

$$
\begin{aligned}
\varrho= & \left(\ell_{0}, v_{0}\right) \stackrel{\delta_{0}}{\longrightarrow}\left(\ell_{0}, v_{0}+\delta_{0}\right) \stackrel{a_{0}}{\longrightarrow}\left(\ell_{1}, v_{1}\right) \cdots \\
& \cdots \stackrel{a_{n-1}}{\longrightarrow}\left(\ell_{n}, v_{n}\right) \stackrel{\delta_{n}}{\longrightarrow}\left(\ell_{n}, v_{n}+\delta_{n}\right)
\end{aligned}
$$

be a run of $A$. We denote by $e_{i}=\left(\ell_{i},\left(g_{i}, a_{i}, R_{i}\right), \ell_{i+1}\right)$ the discrete transition taken from $\left(\ell_{i}, v_{i}+\delta_{i}\right)$ to $\left(\ell_{i+1}, v_{i+1}\right)$. 
The cost of the run $\varrho$ is defined by:

$$
\operatorname{Cost}(\varrho)=\Sigma_{i \in 0 . . n} \operatorname{Cost}\left(\ell_{i}\right) \cdot \delta_{i}+\Sigma_{i \in 0 . . n-1} \operatorname{Cost}\left(e_{i}\right) .
$$

The mean cost of $\varrho$ is defined to be the cost per time unit and given ${ }^{4}$ by $\overline{\operatorname{Cost}}(\varrho)=\operatorname{Cost}(\varrho) / \operatorname{Dur}(\varrho)$. The cost of runs of duration $t \in \mathbb{R}_{>0}$ is defined by $\overline{\operatorname{Cost}}(t)=$ $\sup \{\overline{\operatorname{Cost}}(\llbracket O b s \rrbracket(\varrho)) \mid \operatorname{Dur}(\varrho)=t\}$. The maximal mean cost of $(A, \operatorname{Cos} t)$ is $\overline{\operatorname{Cost}}(A)=\lim \sup _{t \rightarrow \infty} \overline{\operatorname{Cost}}(t)$. The minimal mean cost is defined dually and denoted $\operatorname{Cost}(A)$.

\section{B. Cost of an Observer}

To select a best or optimal dynamic observer which ensures $\Delta$-diagnosability, we need to define a metric to compare them. We extend the one defined in [3] for DES to take into account (real) time elapsing.

Let $A$ be a TA and $O b s$ a DTA observer. $O b s$ is extended into a $\mathrm{P}(\mathrm{D}) \mathrm{TA}$ by associating costs with locations and transitions. The cost associated with the discrete transitions is the cost of switching on the sensors for a set of observable events, and the cost of a location is the cost per time unit of having a set of sensors activated.

Let $\varrho$ be a run of $A$. As $O b s$ is deterministic (and complete) there is exactly one run of $O b s$ the trace of which is $\llbracket O b s \rrbracket(\operatorname{tr}(\varrho))$. Given $\varrho$, let $\llbracket O b s \rrbracket(\varrho)$ be this unique run. The average cost of the run $\varrho$ observed by $O b s$ is $\overline{\operatorname{Cost}}(\llbracket O b s \rrbracket(\varrho))$.

Given $t \in \mathbb{R}_{>0}$, the maximal mean cost of runs of duration $t$ is defined by:

$$
\overline{\operatorname{Cost}}(A, \text { Obs }, t)=\sup _{\varrho \in \text { Runs }^{*}(A) \wedge \operatorname{Dur}(\varrho)=t}\{\overline{\operatorname{Cost}}(\llbracket O b s \rrbracket(\varrho))\} .
$$

The maximal average cost of the pair $\langle A, O b s\rangle$ is defined

$$
\overline{\operatorname{Cos} t}(<A, \text { Obs }>)=\limsup _{t \rightarrow \infty} \overline{\operatorname{Cost}}(A, \text { Obs }, t) .
$$

We can then state the following problem:

Problem 9 (Cost of an Observer):

InPUTS: A TA $A$ and $(O b s, C o s t)$ a PDTA observer.

Problem: Compute $\overline{\operatorname{Cost}}(<A, O b s>)$.

\section{Computing the Cost of a Given Timed Observer}

The computation of optimal infinite schedules for TA has been addressed in [19]. The main result of [19] is:

Theorem 7 (Minimal/Maximal Mean Cost [19]): Given a PTA $A$, computing $\overline{\text { Cost }}$ and $\underline{\text { Cost }}$ is PSPACE-complete. The definition of the cost of an observer is exactly the definition of the maximal mean cost in [19] and thus:

Theorem 8: Problem 9 is PSPACE-complete.

Proof: PSPACE-easiness follows from Theorem 77 note that Theorem 7 assumes that the TA is bounded which is not a restriction as every TA can be transformed into an equivalent (timed bisimilar) bounded TA. For PSPACEhardness, to compute the maximal mean cost of a PDTA $B$, let $A$ be the universal automaton on the alphabet of $B$. Consider $B$ as an observer and solve Problem 9 This solves the maximal mean cost computation problem for DTA. This completes the hardness proof.

\footnotetext{
${ }^{4}$ Runs of duration 0 are not taken into account.
}

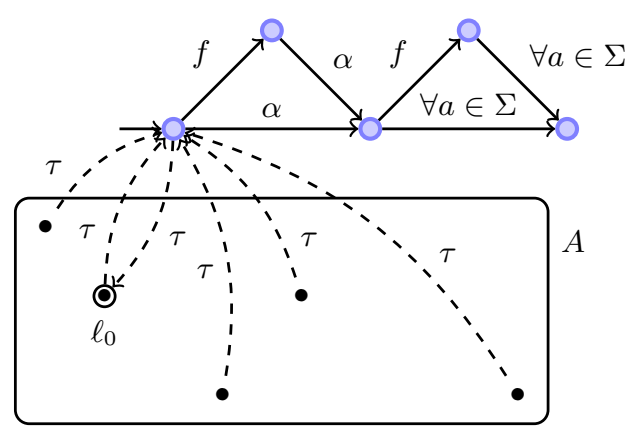

Figure 2. Automaton $B$

\section{Optimal Synthesis Problem}

Checking whether the mean cost of a given observer is less than $k$ requires that we have computed or are given such an observer. A more difficult version of Problem 9 is to check for the existence of cheap dynamic observer:

Problem 10 (Bounded Cost Dynamic Observer):

InPUTS: A TA $A=\left(L, \ell_{0}, X, \Sigma_{\tau, f}, E\right.$, Inv $), \Delta \in \mathbb{N}, \mu$ a resource and $k \in \mathbb{N}$.

PROBLEM:

(A) Is there a dynamic observer $D \in \mathrm{DTA}_{\mu}$ s.t. $A$ is $(D, \Delta)$ diagnosable and $\overline{\operatorname{Cost}}(<A, D\rangle) \leq k$ ?

(B) If the answer to (A) is "yes", compute a witness dynamic observer?

We cannot provide of proof that Problem 10 is decidable. However, we give a lower bound for Problem 10 and later discuss the exact complexity.

Theorem 9: Problem 10 is 2EXPTIME-hard.

Proof: We reduce Problem 8 which is 2EXPTIMEhard [9] to Problem 10. Let $A$ be a TA for which we want to check whether there exists a DTA observer $D \in \mathrm{DTA}_{\mu}$ s.t. $A$ is $(\Delta, D)$-diagnosable.

Let $\alpha$ be a fresh letter not in $\Sigma$. Define the automaton $B$ depicted on Figure 2. The upper part of $B$ generates faulty and non-faulty runs with each letter including $\alpha$. From each location of $A$ (bottom part), we add a $\tau$ transition to the initial state of $B$. The transitions of $A$ are not depicted.

For $B$ to be diagnosable with $\Delta \geq 1$, we must have: 1) $\alpha$ always observable and 2) $\Sigma$ always observable. Moreover, if $A$ is $(\Delta, \Sigma)$-diagnosable, then $B$ is $(\Delta, \Sigma \cup\{\alpha\})$-diagnosable. Conversely, if $B$ is $(\Delta, \Sigma \cup\{\alpha\})$ diagnosable, then $B$ is $(\Delta, \Sigma)$-diagnosable. Hence $A$ is $(\Delta, \Sigma)$-diagnosable iff $B$ is $(\Delta, \Sigma \cup\{\alpha\})$-diagnosable.

Define the cost of the locations to be 1 , and 0 for the transitions in $B . B$ is diagnosable with a DTA $D \in \mathrm{DTA}_{\mu}$ iff there is a dynamic (yet it has to choose $\Sigma \cup\{\alpha\}$ continuously) observer $D$ with $\overline{\operatorname{Cost}}(<A, D\rangle) \leq 1$.

It follows that: there exists a $\mathrm{DTA}_{\mu}$ diagnoser $D$ s.t. $A$ is $(\Delta, \Sigma)$-diagnosable iff $B$ is $(\Delta, O)$-diagnosable with a DTA observer $O \in \mathrm{DTA}_{\mu}$ and $\left.\overline{\operatorname{Cost}}(<A, O\rangle\right) \leq 1$.

The status of Problem 10 is clearly unsettled as the 2EXPTIME-hardness result does not imply it is even decidable. A solution to this problem would be to mimic the one given for DES [3]: solve a mean payoff timed game with 
TABLE I

SUMMARY OF THE RESULTS

\begin{tabular}{||c|c|c|c||}
\hline \hline & \multirow{2}{*}{$\begin{array}{c}\text { Static Observers } \\
\text { Min. Cardinality }\end{array}$} & \multicolumn{2}{|c||}{ Dynamic Observers } \\
\cline { 3 - 4 } & Most Perm. Obs. & Optimal Observer \\
\hline \hline DES & NP-Complete [1] & 2EXPTIME [1] & 2EXPTIME [2] \\
\hline TA & PSPACE-Complete & 2EXPTIME & 2EXPTIME-hard \\
\hline \hline
\end{tabular}

a counterpart of Zwick and Paterson algorithm [20] using the most permissive observers obtained in section $\mathrm{V}-\mathrm{E}$. The type of priced timed games we would have to solve has the following features: 1) they are turn-based, as one Player picks up (controllable moves) a set of events to be observed and then hands it over to the other Player who tries to produce a confusing ${ }^{5}$ run (uncontrollable moves); 2) they have at least two clocks (one for the system $A$ and one for the DTA observer); 3) the controllable choices are urgent i.e., no time can elapse in Player 1 locations. We denote S-PTGA for the class of timed game automata previously defined.

Unfortunately, there is no counterpart of the general result of Zwick \& Paterson for timed automata. Only very few results are known for timed mean payoff games [21], [22], [23], [24] and none of them can be used in our setting. Nevertheless, due to the particular nature of the mean payoff price timed game we construct (in the class S-PTGA), we might be able to compute the optimal choices of observable events using an algorithm similar to [19]. Hence we could obtain a 2EXPTIME algorithm for Problem 10.

\section{CONCLUSION}

The results of the paper are summarized by the line "TA" in Table I The complexity/decidability status of Problem 10 is left open. A solution to this problem would be to solve the following optimization problem on the class of S-PTGA:

\section{Problem 11 (Optimal Infinite Schedule in S-PTGA):}

InPUTS: A S-PTGA $(A$, Cost $)$, a set of Bad states and $k \in \mathbb{N}$. PRoblem: Is there a strategy $f$ for Player 1 in $A$ s.t. $f(A)(A$ controlled by $f$ ) avoids Bad and satisfies $\overline{\operatorname{Cost}}(f(A)) \leq k$ ?

\section{REFERENCES}

[1] F. Cassez, S. Tripakis, and K. Altisen, "Sensor minimization problems with static or dynamic observers for fault diagnosis," in 7th Int. Conf. on Application of Concurrency to System Design (ACSD'07). IEEE Computer Society, 2007, pp. 90-99.

[2] — "Synthesis of optimal dynamic observers for fault diagnosis of discrete-event systems," in Proceedings of the 1st IEEE \& IFIP International Symposium on Theoretical Aspects of Software Engineering (TASE'07). IEEE Computer Society, 2007, pp. 316-325.

[3] F. Cassez and S. Tripakis, "Fault diagnosis with static and dynamic diagnosers," Fundamenta Informaticae, vol. 88, no. 4, pp. 497-540, Nov. 2008.

[4] P. Ramadge and W. Wonham, "Supervisory control of a class of discrete event processes," SIAM Journal of Control and Optimization, vol. 25, no. 1, pp. 1202-1218, 1987.

[5] M. Sampath, R. Sengupta, S. Lafortune, K. Sinnamohideen, and D. Teneketzis, "Diagnosability of discrete event systems," IEEE Transactions on Automatic Control, vol. 40, no. 9, Sept. 1995.

${ }^{5}$ Which is the trace of both a faulty and non-faulty run.
[6] S. Jiang, Z. Huang, V. Chandra, and R. Kumar, "A polynomial algorithm for testing diagnosability of discrete event systems," IEEE Transactions on Automatic Control, vol. 46, no. 8, Aug. 2001.

[7] T.-S. Yoo and S. Lafortune, "Polynomial-time verification of diagnosability of partially-observed discrete-event systems," IEEE Transactions on Automatic Control, vol. 47, no. 9, pp. 1491-1495, Sept. 2002.

[8] S. Tripakis, "Fault diagnosis for timed automata," in Proceedings of the International Conference on Formal Techniques in Real Time and Fault Tolerant Systems (FTRTFT'02), ser. LNCS, W. Damm and E.-R. Olderog, Eds., vol. 2469. Springer Verlag, 2002, pp. 205-224.

[9] P. Bouyer, F. Chevalier, and D. D'Souza, "Fault diagnosis using timed automata," in FoSSaCS, ser. LNCS, V. Sassone, Ed., vol. 3441. Springer Verlag, 2005, pp. 219-233.

[10] R. Alur and D. Dill, "A theory of timed automata," Theoretical Computer Science, vol. 126, pp. 183-235, 1994.

[11] F. Cassez, "A Note on Fault Diagnosis Algorithms," in 48th IEEE Conference on Decision and Control and 28th Chinese Control Conference. Shanghai, P.R. China: IEEE Computer Society, Dec. 2009.

[12] R. Cieslak, C. Desclaux, A. Fawaz, and P. Varaiya, "Supervisory control of discrete-event processes with partial observations," IEEE Transactions on Automatic Control, vol. 33, pp. 249-260, 1988.

[13] P. Bouyer, D. D'Souza, P. Madhusudan, and A. Petit, "Timed control with partial observability," in Proceedings of the 15th International Conference on Computer Aided Verification (CAV'03), ser. LNCS, W. A. Hunt, Jr and F. Somenzi, Eds., vol. 2725. Boulder, Colorado, USA: Springer, July 2003, pp. 180-192.

[14] O. Finkel, "On decision problems for timed automata," Bulletin of the European Association for Theoretical Computer Science, vol. 87, pp. 185-190, 2005

[15] S. Tripakis, "Folk theorems on the determinization and minimization of timed automata," Information Processing Letters, vol. 99, no. 6, pp. 222-226, 2006.

[16] R. Alur, L. Fix, and T. A. Henzinger, "A determinizable class of timed automata," in Proceedings of the 6th International Conference on Computer Aided Verification (CAV'94), ser. LNCS, vol. 818. Springer Verlag, 1994, pp. 1-13.

[17] G. Behrmann, A. Fehnker, T. Hune, K. G. Larsen, P. Pettersson, J. Romijn, and F. Vaandrager, "Minimum-cost reachability for priced timed automata," in Proc. 4th International Workshop on Hybrid Systems: Computation and Control (HSCC'01), ser. LNCS, vol. 2034. Springer, 2001, pp. 147-161.

[18] R. Alur, S. La Torre, and G. J. Pappas, "Optimal paths in weighted timed automata," in Proc. 4th Int. Work. Hybrid Systems: Computation and Control (HSCC'O1), ser. LNCS, vol. 2034. Springer, 2001, pp. 49-62.

[19] P. Bouyer, E. Brinksma, and K. G. Larsen, "Optimal infinite scheduling for multi-priced timed automata," Formal Methods in System Design, vol. 32, no. 1, pp. 2-23, Feb. 2008.

[20] U. Zwick and M. Paterson, "The complexity of mean payoff games on graphs," Theoretical Computer Science, vol. 158, no. 1-2, pp. 343 359, 1996.

[21] P. Bouyer, K. G. Larsen, N. Markey, and J. I. Rasmussen, "Almost optimal strategies in one-clock priced timed automata," in Proceedings of the 26th Conference on Foundations of Software Technology and Theoretical Computer Science (FSTTCS'06), ser. LNCS, N. Garg and S. Arun-Kumar, Eds., vol. 4337. Kolkata, India: Springer, Dec. 2006, pp. 345-356.

[22] P. Bouyer, U. Fahrenberg, K. G. Larsen, N. Markey, and J. Srba, "Infinite runs in weighted timed automata with energy constraints," in Proceedings of the 6th International Conference on Formal Modelling and Analysis of Timed Systems (FORMATS'08), ser. LNCS, F. Cassez and C. Jard, Eds., vol. 5215. Saint-Malo, France: Springer, Sept. 2008, pp. 33-47.

[23] P. Bouyer, Th. Brihaye, M. Jurdziński, R. Lazić, and M. Rutkowski, "Average-price and reachability-price games on hybrid automata with strong resets," in Proceedings of the 6th International Conference on Formal Modelling and Analysis of Timed Systems (FORMATS'08), ser. LNCS, F. Cassez and C. Jard, Eds., vol. 5215. Saint-Malo, France: Springer, Sept. 2008, pp. 63-77.

[24] P. Bouyer, U. Fahrenberg, K. G. Larsen, and N. Markey, "Timed automata with observers under energy constraints," in Proceedings of the 13th International Conference on Hybrid Systems: Computation and Control (HSCC'10), K. H. Johansson and W. Yi, Eds. Stockholm, Sweden: ACM Press, Apr. 2010, to appear. 